\title{
Perfect pitch reconsidered
}

\author{
Author: Calum Moulton ${ }^{\mathrm{A}}$
}

Perfect pitch, or absolute pitch (AP), is defined as the ability to identify or produce the pitch of a sound without need for a reference pitch, and is generally regarded as a valuable asset to the musician. However, there has been no recent review of the literature examining its aetiology and its utility taking into account emerging scientific advances in AP research, notably in functional imaging. This review analyses the key empirical research on AP, focusing on genetic and neuroimaging studies. The review concludes that: AP probably has a genetic predisposition, although this is based on limited evidence; early musical training is almost certainly essential for AP acquisition; and, although there is evidence that it may be relevant to speech processing, AP can interfere with relative pitch, an ability on which humans rely to communicate effectively. The review calls into question the value of AP to musicians and nonmusicians alike.

KEYWORDS: Absolute pitch, early learning model, relative pitch, tonal language, fixed-do training

\section{What is it?}

Perfect pitch, or absolute pitch (AP), is generally defined as the ability to identify or produce the pitch of a sound, without the need for a reference pitch. The criteria for AP possession are poorly defined but most scientists view AP as a spectrum of ability, which ranges from possessors who can produce any tone without any recent exposure to music, to listeners who can recognise pitches only on a familiar instrument. ${ }^{1}$ It is generally regarded as a valuable asset to the musician, which has led to decades of research into its aetiology.

\section{Is it a product of nature or nurture?}

Two rival models were proposed for AP acquisition. An 'early learning' theory assumes a critical period for AP acquisition. In 1901, Abraham proposed a variant on the early learning theory, the 'unlearning of AP' viewpoint, which argued that, because most early musical experience is not conducive to AP development, a widely prevalent inborn potential for developing

Author: ${ }^{A}$ CT3 in psychiatry, South London and Maudsley NHS Foundation Trust London, UK and NIHR academic clinical fellow, Institute of Psychiatry, King's College London, UK
AP is trained out of most people. ${ }^{2}$ Conversely a 'giftedness' model treats AP as a genetic trait that is either inherited or not inherited. ${ }^{3}$ The correlation between early training and development of AP is corroborated by numerous studies. A survey of over 600 music college students found that $40 \%$ of respondents who had begun their musical training before the age of 4 years developed AP, whereas only $3 \%$ of those beginning after the age of 9 years acquired AP. ${ }^{4}$

It has been argued that 'AP is one of the cleanest examples of a human cognitive ability that arises from the interaction of genetic factors and environmental input during development'. Of the AP possessors in the above study, $48 \%$ indicated that they had first-degree relatives who also possessed AP, whereas only $14 \%$ of the non-AP possessors reported first-degree relatives with $\mathrm{AP}^{4}$ The authors argue that this may indicate that a genetic mechanism is involved in the development of AP. These findings could, however, conversely indicate that AP possessors, with their increased likelihood of having experienced early musical training, are more likely to have parents who expose their other children to similar environments. A later study by the same authors negates the environmental component more effectively by discounting anyone who started training after the age of 6 years. ${ }^{6}$

Other studies have taken this a step further by differentiating between different types of musical training: 'fixed-do' training methods, such as the Yamaha method, associate solfège syllables (do, re, mi) with particular standard pitches; these associations do not change. 'Moveable-do' techniques, such as the Suzuki method, associate solfège syllables with a scalar function within a key, such that 'do' can associate with different pitches. This emphasises the development of relative pitch ability, and seems to contradict the common perception that the Suzuki method encourages AP development. Looking only at people who had received 'fixed-do' training before the age of 7 years, one study showed that siblings of AP possessors were 19 times more likely to have AP themselves than siblings of non-AP possessors. ${ }^{7}$ Eliminating differences in musical training does not, however, negate the environmental component; siblings will experience similar environments aside from musical training.

Despite the weight of evidence supporting the early learning model, most music college students who began music lessons at, or before, 6 years of age did not exhibit AP. ${ }^{4}$ Furthermore, a more recent twin analysis study of AP possessors found casewise concordance rates of $78.6 \%$ for monozygotic twins and $45.2 \%$ for dizygotic twins, although these findings are based on small sample numbers and rely on the self reporting 
of twin status and their AP ability. ${ }^{8}$ Segregation analysis by the same authors found a segregation ratio of 0.089 , indicating that any genetic component appears to be polygenic in nature.

Some authors have proposed findings from neuroimaging research as further evidence for innate factors in AP acquisition. Schlaug et al found that AP possessors exhibited a greater leftward asymmetry of the planum temporale (PT) in the temporal lobe, a region central to speech processing. ${ }^{9}$ The authors subsequently found that early beginning non-AP musicians did not exhibit the exaggerated leftward PT asymmetry of their AP counterparts, arguing that this asymmetry may be a determinant of AP acquisition rather than a consequence. ${ }^{10}$ However, the average age of AP possessors in this study was 27 years, making it difficult to distinguish innate factors from environmental factors so many years after AP acquisition. Stronger evidence would require study of much younger AP possessors, which would negate environmental confounding better. The ideal 'control' group would be a group of AP possessors who did not receive early musical training, but, for the reasons above, this is not likely to be feasible.

In summary, some people appear to have a polygenic genetic predisposition to AP development but the relative contribution of this is unknown and good quality research is needed in this area. Early musical training is almost certainly essential for AP acquisition, although some methods of musical training are much more conducive to AP development than others.

\section{Does it make you a better musician?}

AP is generally regarded as useful to a musician, for example as an aid to intonation or sight singing. However, experimental evidence shows that some AP possessors have considerable difficulty in identifying pitch relations in transposed tonal contexts. ${ }^{11}$

Some authors argue that, when identifying musical intervals, most musicians directly evaluate the musical interval between two notes: the relative pitch (RP) strategy. ${ }^{12}$ AP possessors, however, in addition to the RP strategy, can first identify the two pitches and then infer the musical interval between

\section{Key points}

> Absolute pitch is defined as the ability to identify or produce the pitch of a sound, without the need for a reference pitch

> Early musical training seems to be essential for absolute pitch development, though the nature of this training is important

> There is probably a genetic component to absolute pitch, although much further research is needed in this area

$>$ Neuroimaging studies suggest that absolute pitch possessors neglect the ability of relative pitch

> Prevalence of absolute pitch is higher in speakers of tona languages them: the AP strategy. In functional brain imaging studies, AP possessors show increased activation of the left superior temporal sulcus during the early encoding phase of a pitch memory task, an area shown to be involved in categorisation tasks. ${ }^{13}$ The increased activation seen in the right superior parietal lobule and intraparietal sulcus in non-AP possessors may reflect the use of a visuospatial mapping scheme, such as imaging notes on a stave or using spatial coding for their relative pitch height. ${ }^{13}$

AP possessors show strong activation of the left posterior dorsolaterofrontal cortex in response to isolated tones, a response not demonstrated in non-AP listeners. ${ }^{14}$ The posterior dorsolaterofrontal cortex was also implicated in a study in which non-musicians were trained to associate chords with arbitrary numbers. ${ }^{15}$ This area has been implicated in conditional associative learning of sensory stimuli, such as the association between the pitch of a sound and a verbal label. During an interval-judgement task, non-AP possessors showed activity within the right inferior frontal cortex not demonstrated in AP possessors. This implicates the right inferior frontal cortex in the maintenance of pitch information in auditory tonal working memory. The authors conclude that AP possessors may compute the size of the musical interval by knowing what the individual notes are, rather than by maintaining pitch in working memory. ${ }^{14}$

It has been suggested that some AP possessors do not learn to develop relative pitch in its fullness, because they have learned to rely on AP in their musical activities. ${ }^{11}$ Perhaps, then, some AP possessors try to perform interval recognition tasks using the RP strategy, but their comparatively poor sense of RP leads to poorer performance than possessors of RP alone. In a study looking at multi-channel event-related potentials, people with different levels of AP underwent a pitch-naming task, where they were asked to report vocally the pitches of stimuli after hearing a cue, and also a listening task, where they were asked to respond by vocalising the syllable 'ah' to all stimuli regardless of their pitches. Naming-minus-listening difference waves were marked in people with lower levels of AP, suggesting use of RP in the pitch-naming task. Conversely, high-AP possessors showed less difference, suggesting that AP possessors name pitches covertly and automatically on hearing tones, even when this is not necessary. ${ }^{16}$ In an auditory stroop functional magnetic resonance imaging (fMRI) experiment, AP possessors performed better than non-AP musicians on a congruent sequence (where frequencies of tones corresponded to syllables that were the names of the scale tones) but no better on an incongruent sequence. Furthermore, the left anterosuperior temporal gyrus/sulcus region was activated in AP musicians only for the detection of verbal/tonal incongruencies, suggesting that this region in AP musicians is involved in perceiving and identifying tones. ${ }^{17}$

The most likely explanation for these findings, the author believes, is that an AP possessor's performance depends on how they use this ability. The balance of evidence suggests that the tone-label associative mechanism is engaged automatically when an AP possessor hears a tone, but that many AP possessors do not use this information to identify the interval. This latter ability requires RP and, just as acquisition of AP requires early musical training, a good sense of RP probably requires years of teaching about the relative features of music. 
In this respect, non-AP possessors are entitled to view their AP possessor colleagues as musical equals.

\section{Does it matter?}

The relationship between AP and RP seems uneasy, and this has more fundamental implications beyond music. Western languages such as English use pitch syntactically to convey expression, such as raising the tone of the voice at the end of the sentence to indicate a question. The idea of a fixed tone then seems somewhat incongruous in a species that relies on RP for communication. Some animals, by contrast, do use AP to communicate with others. Experiments on songbirds found that RP perception, although within the birds' capacity, was subordinate to stimulus processing based on $\mathrm{AP}^{18}$

Only recently has credible evidence emerged that a fundamental role for AP may not be confined to the animal kingdom. Diana Deutsch showed that speakers of tonal languages, such as Mandarin and Vietnamese, when asked to recite words on different days, do so at very similar pitches, a pattern not demonstrated among English speakers. ${ }^{19,20}$ Moreover, recent studies have shown that Cantonese speakers outperform their English-speaking non-musician counterparts on various measures of pitch and music perception. ${ }^{21}$

Studies have demonstrated significantly higher prevalence of AP among Asian students than their white counterparts, despite no difference in frequency of early music exposure. ${ }^{7}$ Asian students, however, were significantly more likely to have received 'fixed-do' training than white students. Deutsch hypothesises that AP for music might then be acquired by tone language speakers in the same way as they would acquire the pitches of a second tonal language. ${ }^{22}$ A more recent study demonstrated significantly higher AP prevalence among fluent tonal language speakers than non-fluent tonal language speakers, thereby questioning the role of ethnicity as a predisposing factor in AP acquisition. However, the numbers in the study are small, and the type of early musical training fixed-do or non-fixed-do - is again not analysed. ${ }^{23}$

There is also recent fMRI evidence that AP may be relevant to speech processing. In response to variations in the lexical and/or prosodic information of speech stimuli, AP possessors showed stronger activations in the posterior part of the middle temporal gyrus, an area involved in higher-order language processing, as well as stronger left-lateralised activation of the superior temporal sulcus during processing of segmental speech information. ${ }^{24}$ The authors propose, therefore, that the auditory acuity of AP may extend beyond musical processing to a more general notion of acoustic segmentation by fully integrating left-hemispheric, speech-relevant networks.

The hypothesis that tonal language speakers use AP to aid communication is potentially exciting, because it could provide the missing link as to why AP should provide any fundamental value to humans at all. However, these findings must first be adjusted for exposure to fixed-do musical training, before the theory that AP originated as a feature of speech can gain better credence. Even if true, the continued persistence of AP in the west remains an enigma. For now, absolute pitch - a trait of questionable value to musicians and non-musicians alike seems little more than an atavism.

\section{References}

1 Levitin D, Rogers S. Absolute pitch: perception, coding and controversies. Trends Cogn Sci 2005;9:26-33.

2 Abraham O. Das absolute Tonbewusstsein. Sammelbände der Internationalen Musikgesellschaft 1901;3:1-86.

3 Bachem A. Various types of absolute pitch. J Acoust Soc Am 1937;9:146-51.

4 Baharloo S, Johnston P, Service S et al. Absolute pitch: An approach for identification of genetic and nongenetic components. Am J Hum Genet 1998;2:224-31.

5 Zatorre R. Absolute pitch: a model for understanding the influence of genes and development on neural and cognitive function. Nat Neurosci 2003;6:692-5.

6 Baharloo S, Service S, Risch N et al. Familial aggregation of absolute pitch. Am J Hum Genet 2000;67:755-8.

7 Gregersen P, Kowalsky E, Kohn N, Marvin E. Early childhood music education and predisposition to absolute pitch: teasing apart genes and environment. Am J Med Genet 2001;98:280-2.

8 Theusch E, Gitschier J. Absolute pitch twin study and segregation analysis. Twin Res Human Genet 2011;14:173-8.

9 Schlaug G, Jäncke L, Huang Y, Steinmetz H. In vivo evidence of structural brain asymmetry in musicians. Science 1995;267:699-701.

10 Keenan JP, Thangaraj V, Halpern AR, Schlaug G. Absolute pitch and planum temporale. Neuroimage 2001;14:1402-8.

11 Miyazaki K. Absolute pitch as an inability: identification of musical intervals in a tonal context. Music Percept 1993;11:55-72.

12 Takeuchi A, Hulse S. Absolute pitch judgments of black- and whitekey pitches. Music Percept 1991;9:27-46.

13 Schulze K, Gaab N, Schlaug G. Perceiving pitch absolutely: comparing absolute and relative pitch possessors in a pitch memory task. BMC Neurosci 2009; 10:106.

14 Zatorre R, Perry D, Beckett C et al. Functional anatomy of musical processing in listeners with absolute pitch and relative pitch. Proc Natl Acad Sci USA 1998;95:3172-7.

15 Bermudez P, Zatorre RJ. Conditional associative memory for musical stimuli in nonmusicians: implications for absolute pitch. J Neurosci 2005;25:7718-23.

16 Itoh K, Suwazono S, Arao H et al. Electrophysiological correlates of absolute pitch and relative pitch. Cereb Cortex 2005;15:760-9.

17 Schulze K, Mueller K, Koelsch S. Auditory stroop and absolute pitch: an fMRI study. Hum Brain Mapp 2013;34:1579-90.

18 Hulse S, Cynx J. Relative pitch perception is constrained by absolute pitch in songbirds (Mimus, Molothrus, and Sturmus). J Com Psychol 1985;99:176-96.

19 Deutsch D, Henthorn T, Dolson M. Absolute pitch is demonstrated in speakers of tone languages. J Acoust Soc Am 1999;106;2267.

20 Deutsch D. The enigma of absolute pitch. Acoustics Today 2006;2:11-19.

21 Bidelman GM, Hutka S, Moreno S. Tone language speakers and musicians share enhanced perceptual and cognitive abilities for musical pitch: evidence for bidirectionality between the domains of language and music. PLOS One 2013;8:e6067.

22 Deutsch D, Henthorn T, Marvin E, Xu H. Perfect pitch in tone language speakers carries over to music. J Acoust Soc Am 2005;116:2580.

23 Deutsch D, Dooley K, Henthorn T, Head B. Absolute pitch among students in an American music conservatory: Association with tone language fluency. J Acoust Soc Am 2009;125:2398-403.

24 Oechslin MS, Meyer M, Jäncke L. Absolute pitch - functional evidence of speech-relevant auditory acuity. Cereb Cortex 2010;20:447-55.

Address for corresponding author: Dr C Moulton, Department of Psychological Medicine, Institute of Psychiatry, King's College London, 10 Cutcombe Road, London SE5 9RJ.

Email: calum.moulton@kcl.ac.uk 